
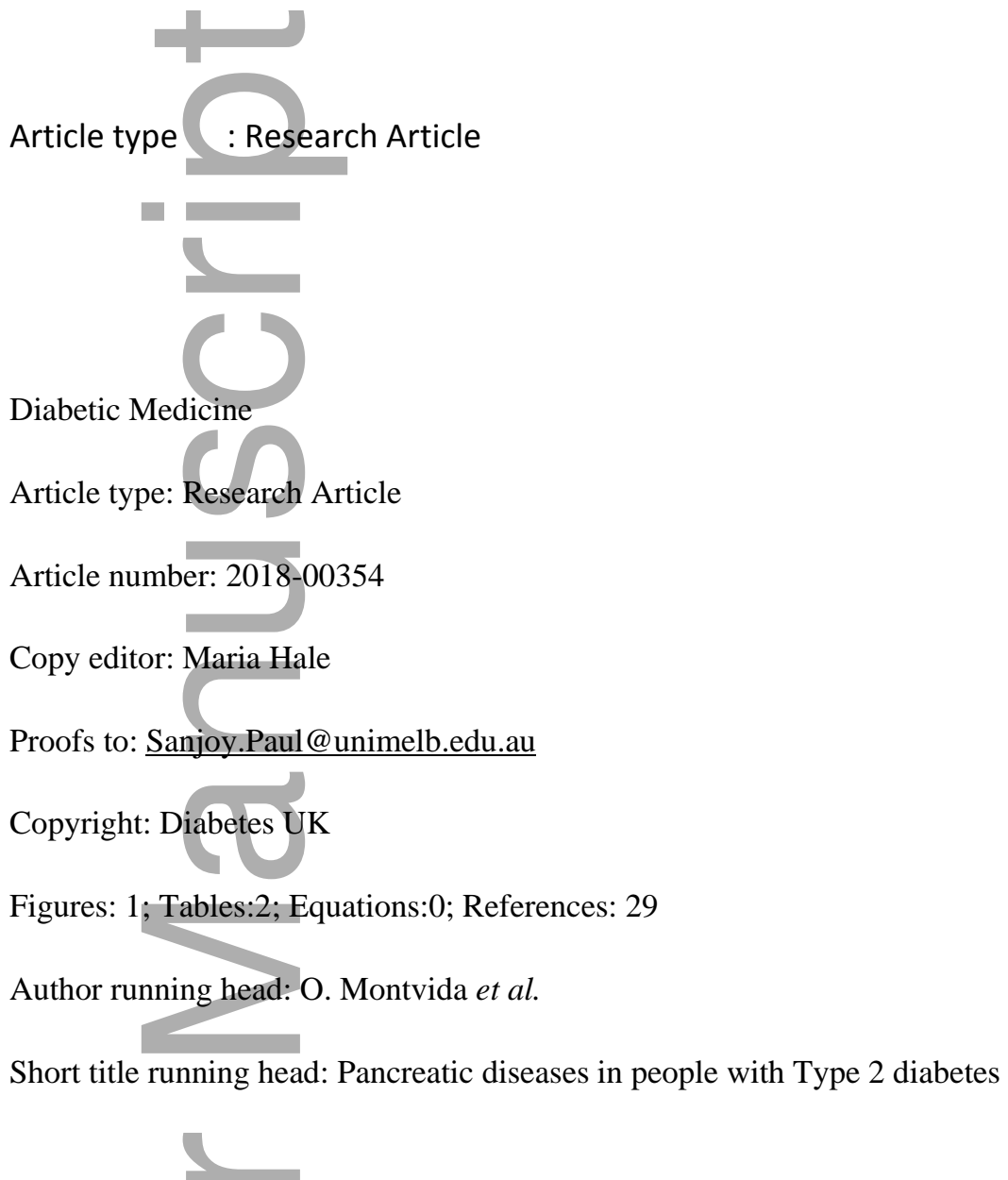

Research Article: Treatment

\title{
Treatment with incretins does not increase the risk of pancreatic diseases compared to older anti- hyperglycaemic drugs, when added to metformin: real world evidence in people with Type 2 diabetes
}

This is the author manuscript accepted for publication and has undergone full peer review but has not been through the copyediting, typesetting, pagination and proofreading process, which may lead to differences between this version and the Version of Record. Please cite this article as doi: $10.1111 /$ dme.13835

This article is protected by copyright. All rights reserved 


\title{
O. Montvida ${ }^{1,2}$ J. B. Green ${ }^{3}$ J. Atherton ${ }^{4}$ and S. K. Paul ${ }^{1,5}$
}

\author{
${ }^{1}$ Statistics Unit, QIMR Berghofer Medical Research Institute and ${ }^{2}$ School of Biomedical Sciences, Queensland \\ University of Technology, Brisbane, Australia, ${ }^{3}$ Division of Endocrinology and Duke Clinical Research \\ Institute, Duke University Medical Center, Durham, NC, USA, ${ }^{4}$ Cardiology Department, Royal Brisbane and \\ Women's Hospital and University of Queensland School of Medicine, Brisbane and ${ }^{5}$ Melbourne EpiCentre, \\ University of Melbourne and Melbourne Health, Melbourne, Australia \\ Correspondence to: Sanjoy Ketan Paul. E-mail: $\underline{\text { Sanjoy.Paul@unimelb.edu.au }}$
}

\begin{abstract}
Aims In people with metformin-treated diabetes, to evaluate the risk of acute pancreatitis, pancreatic cancer and other diseases of the pancreas post second-line anti-hyperglycaemic agent initiation.
\end{abstract}

Methods People with Type 2 diabetes diagnosed after 2004 who received metformin plus a dipeptidyl peptidase-4 inhibitor (DPP-4i, $n=50$ 095), glucagon-like peptide-1 receptor agonist (GLP-1RA, $n=12$ 654), sulfonylurea $(n=110747)$, thiazolidinedione $(n=17597)$ or insulin $(n=34805)$ for at least 3 months were identified in the US Centricity Electronic Medical Records. Time to developing acute pancreatitis, other diseases of the pancreas and pancreatic cancer was estimated, balancing and adjusting anti-hyperglycaemic drug groups for appropriate confounders.

Results In the DPP-4i group, the adjusted mean time to acute pancreatitis was 2.63 [95\% confidence intervals $(\mathrm{CI}) 2.38,2.88$ ] years; time to pancreatic cancer was $2.70(2.19,3.21)$ years; and time to other diseases of the pancreas was $2.73(2.33,3.12)$ years. Compared with DPP-4i, the insulin group developed acute pancreatitis 0.48 years $(P<0.01)$ earlier and the GLP-1RA group developed pancreatic cancer 3 years later $(P<0.01)$. However, with the constraint of no event within 6 months of insulin initiation, the risk of acute pancreatitis in the insulin group was insignificant. No other significant differences were observed between groups.

Conclusions No significant differences in the risk of developing pancreatic diseases in those treated with various anti-hyperglycaemic drug classes were found. 


\section{What's new?}

- Association of treatment with incretin-based therapies and the risk of pancreatic diseases remains controversial. However, no study explored the comparative safety of different anti-hyperglycaemic drugs in this context.

- This study provides a holistic population-level comparative outcome evaluation of the risk of pancreatic diseases from the time of receiving different second-line antihyperglycaemic drugs post metformin.

- Although treatment with incretin-based therapies was not found be to be associated with an increased risk of pancreatic diseases, people treated with insulin experienced higher risk of such diseases.

\section{$<$ H1 $>$ Introduction}

Glucagon-like peptide-1 receptor agonists (GLP-1RAs) and dipeptidyl peptidase-4 inhibitors (DPP-4i) represent incretin-based therapeutic drug classes used to treat Type 2 diabetes. These drugs have demonstrated efficacy in reducing blood glucose levels with low risk of hypoglycaemia [1-3]. Treatment with GLP-1RAs is associated with favourable changes in metabolic measurements such as body weight, and some agents in the class have been shown to reduce the risk of cardiovascular events [3-5]. However, some recent clinical observational studies have raised questions as to the possible association of treatment with incretin-based therapies, particularly with DPP-4i, and the risk of acute pancreatitis or pancreatic cancer [617].

Although a number of cohort studies and meta-analyses reported no association between incretin-based therapies and risk of acute pancreatitis and pancreatic cancer $[9,12,13,15,16,18]$, other studies have reported an increased risk of acute pancreatitis with such agents $[8,11,14,17]$. In a meta-analysis based on pooled data from the SAVOR-TIMI 53, EXAMINE and TECOS trials, Tkáč and Raz [8] reported a significant increase in the incidence of acute pancreatitis [odds ratio (OR): 1.79; 95\% confidence intervals (CI) 1.13, 2.82] in people treated with DPP-4i when compared with placebo. The observed increase in the absolute risk of acute pancreatitis with DPP-4i therapy was $0.13 \%$. In a cohort study based on real-world primary care data from the UK, Knapen and colleagues [17] reported a 1.5-fold increased risk of any pancreatitis in incretin-based therapy users compared with 
other non-insulin anti-hyperglycaemic drug users. However, another study by Knapen and colleagues [16], based on the same database, reported no association of incretin-based therapies with the risk of pancreatic cancer.

Previously published cohort studies have generally assessed pancreatic risk by comparing rates of pancreatic diseases in users of incretin-based therapies with rates in users of any nonincretin based anti-hyperglycaemic regimen. However, no prior study of adequate size and duration has holistically evaluated the risks of acute pancreatitis and pancreatic cancer with incretin-based therapy compared with use of other specific anti-hyperglycaemic drug classes. Furthermore, previous publications based on real-world data, in which baseline risks differ significantly and are modified over time by contrasting confounders, may not have utilized optimal analytical approaches to assess risk. Using extensive person-level longitudinal data from ambulatory and primary care systems in the USA, the aims of this exploratory outcome study were to evaluate the rates and risks of developing acute pancreatitis, other diseases of the pancreas or pancreatic cancer in people with metformin-treated Type 2 diabetes who initiated second-line anti-hyperglycaemic therapy with a DPP-4i, GLP-1RA, sulfonylurea, thiazolidinedione or insulin.

\section{$<$ H1 $>$ Materials and methods}

\section{$<$ H2 >Data source}

Centricity Electronic Medical Record (CEMR) of the USA represents a variety of ambulatory and primary care medical practices, including solo practitioners, community clinics, academic medical centres and large integrated delivery networks. Over 35000 physicians and other providers from all US states contribute to the CEMR, wherein $\sim 75 \%$ are primary care providers. The database is generally representative of the US population: the diabetes prevalence ( $7.1 \%$ of people with diabetes identified by diagnostic codes) is similar to the US National Diabetes Statistics (6.7\% diagnosed diabetes in 2014) [19]. CEMR has been used extensively for academic research worldwide [20,21].

For more than 34 million individuals, longitudinal EMRs were available from 1995 to April 2016. This database contains comprehensive person-level information on demographics, anthropometric, clinical and laboratory variables including age, sex, ethnicity, smoking status, and longitudinal measures of body weight, BMI, blood pressure, $\mathrm{HbA}_{1 \mathrm{c}}$, full lipid profiles, urine albumin and creatinine, and serum creatinine. 
Medication data include brand names and doses for individual medications prescribed (RxNorm), along with start/stop dates and specific fields to track treatment alterations. This data set also contains self-reported medications, including prescriptions received outside the EMR network and over-the-counter medications. All disease events along with dates are coded with International Classification of Diseases (ICD)-9, ICD-10 or SNOMED Clinical Terms (CT) codes.

\section{$<$ H2>Study design and study data}

All individuals with a diagnosis of Type 2 diabetes were included in this study with the conditions of no missing data for age, sex or ethnicity; $\geq$ ag8 and $<\quad 80$ years at the diagnosis of Type 2 diabetes; and date of diagnosis of Type 2 diabetes after EMR registration date and after 1 January 2005. All those included also first began anti-hyperglycaemic therapy with metformin, followed by second-line additional treatment with a DPP-4i, GLP1RA, insulin, thiazolidinedione or sulfonylurea for $\geq 3$ months. Users of second-line insulin, thiazolidinedione or sulfonylurea who had ever received a DPP-4i or GLP-1RA were excluded, as were those with other diseases of the pancreas or any type of cancer that occurred prior to initiation of second-line anti-hyperglycaemic drug (index date).

Baseline (index date) data included age, sex, ethnicity, body weight, BMI and blood pressure at the time of second anti-hyperglycaemic drug initiation. Baseline $\mathrm{HbA}_{1 \mathrm{c}}$ was obtained as the closest observation to second drug initiation within a $[-3,+3]$ month window. Body weight, BMI, SBP and lipids were calculated as the average of available measurements within $[-3,+3]$ months of baseline. Obesity was defined as BMI $\geq 30 \mathrm{~kg} / \mathrm{m}^{2}$.

The presence of comorbidities prior/post index date and the time to such events were also obtained. Acute pancreatitis, other diseases of the pancreas, cancer, cardiovascular disease, chronic kidney disease and hypertension were identified. Cancer was defined as any malignant neoplasm or carcinoma in situ. Cancer of the pancreas was additionally separated. Other diseases of the pancreas included specified (e.g. pancreatic cyst) and unspecified diseases of the pancreas with appropriate clinical codes. Cardiovascular disease was defined as ischaemic heart disease (includes myocardial infarction), peripheral vascular/artery disease, heart failure or stroke.

Tobacco use status included data on the use of cigars, pipe, cigarettes, chewing tobacco, snuff and smokeless tobacco. Occasional smokers were classified as 'current'. In case of discordant same-day statuses, priority was given to 'current', rather than to 'former' and lastly to 'never' 
status. Last status recorder prior to index date was preserved as tobacco use status. Complete information on anti-hyperglycaemic drugs, along with non-steroidal anti-inflammatory drugs, lipid-modifying drugs, anti-hypertensive and cardioprotective medications was obtained. Cardioprotective medications included beta-blocking agents, angiotensin-converting enzyme inhibitors, angiotensin II antagonists and statin.

\section{$<$ H2 $>$ Ethical approval}

Research involved existing data, in which individuals could not be identified directly or through identifiers linked to them. Thus, according to the US Department of Health and Human Services Exemption 4 (CFR 46.101(b)(4)), this study is exempt from ethics approval from an institutional review board and informed consent.

\section{$<$ H2>Statistical methods}

All analyses were performed by class of second-line anti-hyperglycaemic drugs. Basic statistics were presented using number $(\%)$, mean (SD) or median (Q1, Q3), as appropriate. The event rates per 1000 person-years $(95 \% \mathrm{CI})$ were estimated for acute pancreatitis, other diseases of the pancreas, pancreatic cancer using standard life-table method.

In the presence of significant differences in risk factors between comparative treatment groups in observational studies, standard Cox regression survival models after propensity score adjustments are often used. Estimation of hazard ratios are useful for population effects when they are constant, which occurs when the treatment enters linearly, and the distribution of the outcome has a proportional-hazards form [22]. However, decisions on therapeutic introductions and modifications are neither linear nor conform to proportional-hazards form in the context of risk of an event. Given the observational nature of the study, with high likelihood of inherent differences in the comparator treatment groups, we used a 'treatment effect' modelling approach [23-25]. The parametric gamma time-to-event model with inverse probability-weighted regression adjustment for the confounders was used to evaluate the adjusted mean $(95 \% \mathrm{CI})$ time to event for the reference treatment group (DPP-4), and the adjusted time difference $(95 \% \mathrm{CI})$ to the occurrence of event in the comparator treatment groups were estimated. The robustness of choosing gamma distribution was tested on the basis of information criteria estimates. Risk analyses were balanced on age and the follow-up time by treatment groups, and were adjusted for age, sex, smoking status, BMI and diabetes duration, following the weighted propensity-score approach. Survival time was computed as time to event (acute pancreatitis, other diseases of the pancreas or pancreatic cancer) if an 
event occurred, otherwise as time to the end of follow-up (date of a person's last available record within the database). The robustness of risk modelling with balancing factors were evaluated by estimating the weighted standardised differences in these factors by treatment groups.

Two sensitivity analyses were conducted to evaluate the robustness of the risk analyses in two sub-cohorts: (i) in all people from the study cohort excluding those with acute pancreatitis, other diseases of the pancreas or any type of cancer within 6 months of the index date (sub-cohort 1); and (ii) in all people from the study cohort with non-missing baseline $\mathrm{HbA}_{1 \mathrm{c}}$ (sub-cohort 2). Sub-cohort 2 was additionally balanced on $\mathrm{HbA}_{1 \mathrm{c}}$ and body weight for risk analyses.

\section{$<$ H1 $>$ Results}

From 2624954 people identified as having Type 2 diabetes, 225898 met the inclusion criteria for the study (Fig. 1). At the index date, participants had a mean (SD) age of 59 (12) years, $49 \%$ were men, $69 \%$ had White European ancestry, and had an overall mean follow-up time of 3.2 years. Anti-hyperglycaemic drug groups as defined included 22\% $(n=50095)$ using DPP-4i; 6\% $(n=12654)$ using GLP-1RA; 15\% $(n=34805)$ using insulin; $49 \%(n=110747)$ using sulfonylurea; and 8\% $(n=17597)$ using thiazolidinedione (Table 1). Follow-up time of those in most of the treatment groups was similar, except for those in the thiazolidinedione group which had a longer mean follow-up of 4.6 years. The distributions of age, diabetes duration and $\mathrm{HbA}_{1 \mathrm{c}}$ were significantly different between groups, as expected. The proportions of people adding or moving to a third-line antihyperglycaemic drug were similar in those receiving incretin-based therapy (47\% in both the DPP-4i and GLP-1RA groups), although other groups had significantly lower proportions. The non-incretin groups could not have received incretin-based therapies during follow-up, by design.

\section{$<$ H2 $>$ Risk of acute pancreatitis}

Only $1049(0.46 \%)$ people developed acute pancreatitis during the mean 3.2 years of followup (Table 1). The rates per 1000 person-years of acute pancreatitis were similar in the DPP-4i (1.31; 95\% CI 1.21, 1.59), GLP-1RA $(1.49 ; 1.16,1.92)$ and sulfonylurea $(1.45 ; 1.33,1.58)$ groups, whereas those treated with insulin had significantly higher acute pancreatitis rate $(2.01 ; 1.75,2.31)$ and those treated with thiazolidinedione had significantly lower acute pancreatitis rate $(0.89 ; 0.70,1.12)$ compared with DPP-4i group (Table 2$)$. The adjusted mean 
(95\% CI) time to acute pancreatitis in people treated with DPP-4 was $2.63(2.38,2.88)$ years. Those treated with insulin were likely to develop acute pancreatitis 0.48 years $(P<0.01)$ earlier. The adjusted mean time to acute pancreatitis were similar in other treatment groups.

\section{$<$ H2 $>$ Risk of cancer of the pancreas}

In the cohort, $357(0.16 \%)$ people developed cancer of the pancreas, and there was no significant difference in the rate of pancreatic cancer per 1000 person-years among the treatment groups. Among those with acute pancreatitis, 17 (2\%) developed pancreatic cancer, of whom four and two individuals belonged to DPP-4 and GLP-1RA groups, respectively. The adjusted mean $(95 \% \mathrm{CI})$ time to outcome in the DPP-4 group was $2.70(2.19,3.21)$ years, with no significant differences in time to event in the insulin, sulfonylurea and thiazolidinedione groups (Table 2). However, people treated with GLP-1RA were likely to develop pancreatic cancer by $\sim 3$ years later $(P<0.01)$ compared with the DPP-4 group.

\section{$<$ H2 $>$ Risk of other diseases of the pancreas}

Only $0.33 \%(n=752)$ of the cohort experienced other diseases of the pancreas during followup. Among those with acute pancreatitis, $101(10 \%)$ also had other diseases of the pancreas. Except for the insulin group, the rate per 1000 person-years for other diseases of the pancreas was similar across treatment groups (Table 2). The mean $(95 \% \mathrm{CI})$ time to develop other diseases of the pancreas in DPP-4i group was $2.73(2.33,3.12)$ years, with no significant difference from other treatment groups.

In all survival regression models, the balances for defined confounders were achieved among the treatment groups. The weighted standardized differences in confounding factors across the treatment groups were similar while evaluating the risk of acute pancreatitis, other diseases of the pancreas and pancreatic cancer separately (Table S1). The sensitivity analyses with sub-cohorts 1 and 2 showed similar estimates of time to events. However, after removing those who developed acute pancreatitis or other diseases of the pancreas or any cancer within 6 months of the index date (sub-cohort 1), the adjusted time to acute pancreatitis among people treated with insulin was no more different from the other groups, as otherwise observed in the primary cohort.

\section{$<$ H1 $>$ Discussion}

A potential relationship between the use of incretin-based anti-hyperglycaemic drugs and adverse pancreatic outcomes has been suggested by various pre-clinical and clinical studies. 
In particular, meta-analysis of data obtained from cardiovascular safety trials of several DPP4i medications suggests that exposure to those medications significantly increased the risk of acute pancreatitis compared with placebo, although the absolute increase in risk was small [8]. Interestingly, although people with Type 2 diabetes and obesity are known to be at increased risk for pancreatitis and pancreatic cancer, rates of those complications have been low in recent clinical trials. Low rates of these outcomes of interest, as well as the likely extended duration of exposure and follow-up needed to ascertain a relationship between antihyperglycaemic drug exposure and the development of malignancy, pose significant challenges in determining the pancreatic safety of drugs commonly used in diabetes management.

In this retrospective, longitudinal, real-world study of a large cohort of people with metformin-treated Type 2 diabetes, we analysed the effects of second-line antihyperglycaemic drugs upon rates and times to clinically important pancreatic complications. Our analyses have shown that the rate of acute pancreatitis was higher in the group treated with insulin, lower in the thiazolidinedione group, and similar in the groups receiving GLP1RA or sulfonylurea therapy when compared with the group treated with DPP-4i. Time to development of pancreatic cancer was longer in the GLP-1RA group compared with DPP-4i users, but did not differ significantly between the other anti-hyperglycaemic drug groups compared with DPP-4i. Rates of any other disease of the pancreas were not higher among people who received additional therapy with a DPP-4i compared with other classes of antihyperglycaemic drugs.

The increased risk of pancreatitis in insulin users is perhaps not surprising, because insulin users often tend to have a greater burden of comorbidities and risks for adverse outcomes that cannot be fully adjusted for in a retrospective analysis. However, time to acute pancreatitis was no longer significantly different between the insulin and DPP-4i groups after removing individuals who developed acute pancreatitis or other diseases of the pancreas or any cancer within 6 months of the index date (sub-cohort 1). The lower rate per 1000 person-years of acute pancreatitis noted in thiazolidinedione users is perhaps more unexpected. Interestingly, studies in rodent models suggest that rosiglitazone exposure may limit the severity of pancreatitis and shorten recovery from pancreatic inflammation in the setting of induced pancreatic injury [26,27]. However, the thiazolidinedione class is associated with a number of adverse side effects that have significantly limited clinical use of these medications [28]. The findings of this analysis provide reassurance to prescribers and users of DPP-4i that these 
medications do not significantly increase the risk of adverse pancreatic outcomes compared with other commonly prescribed second-line therapies.

Several strengths of this analysis include the large number of people who met the inclusion criteria for analysis, the robust amount of data collected and the reasonable duration of follow-up after addition of the second-line anti-hyperglycaemic drug. Although the overall numbers of people experiencing acute pancreatitis and pancreatic cancer were small, the numbers exceed those available for inclusion in the previously cited meta-analysis [8]. The unique and novel aspects of this study include the careful choice of study cohort without any history of pancreatic diseases or cancer. This approach reduces the likelihood of events attributable to pre-existing conditions such as biliary disease, structural pancreatic disorders or an autoimmune/genetic predisposition to pancreatic diseases. The analyses also include a holistic evaluation of the risks associated with use of different anti-hyperglycaemic drugs rather than a one drug vs. all approach; a detailed evaluation of the treatment patterns, ensuring non-exposure to incretin-based therapies in other comparator treatment groups; and careful choice of statistical methodology to evaluate the risk while robustly addressing the challenging issues of imbalances in important risk factors and confounders across treatment groups. Our finding that people treated with a DPP-4i are not at higher risk of pancreatic diseases is robust, supported by the sensitivity analyses in a large number of people with Type 2 diabetes.

Electronic databases do present challenges in terms of the accuracy and completeness of the required data. As a result, limitations of this study include non-availability of complete and reliable longitudinal data related to medication adherence, tobacco and alcohol consumption, socio-economic status and potential residual confounders. In particular, the inability to quantify alcohol exposure does not permit adjustment or balancing for this known risk factor for pancreatitis. The analyses were not adjusted for conditions such as hypertriglyceridaemia, hypercalcaemia or non-anti-hyperglycaemic drug exposures that have been associated with pancreatitis; however, these are considered responsible for only a small percentage of overall cases of acute pancreatitis [29]. Furthermore, other known risk factors for pancreatic cancer including dietary composition, inactivity or family history/genetic predisposition are not available in routinely collected electronic health records. There may also have been inherent medical, socio-economic or other differences that affected the types of anti-hyperglycaemic drug prescribed. These differences could not be readily determined or adjusted for in the analyses, and the impact of these individual characteristics upon pancreatic outcomes is 
unknown. Future pharmaco-epidemiologic studies with longer-term follow up and more robust medication exposure and outcomes ascertainment will further complement our understanding of the pancreatic safety of anti-hyperglycaemic therapies.

\section{Funding sources}

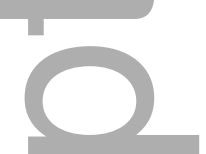

\section{Competing interests}

SKP has acted as a consultant and/or speaker for Novartis, GI Dynamics, Roche, AstraZeneca, Guangzhou Zhongyi Pharmaceutical and Amylin Pharmaceuticals LLC. He has received grants in support of investigator and investigator-initiated clinical studies from Merck, Novo Nordisk, AstraZeneca, Hospira, Amylin Pharmaceuticals, Sanofi-Avensis and Pfizer. JA has received honoraria for consultancies and lectures from Novartis, Novo Nordisk, Astra Zeneca, Sanofi, Merck Sharp and Dohme, Abbott, Janssen Cilag and Takeda. OM has no conflict of interest to declare. JG has received research grants from AstraZeneca, Boehringer Ingelheim, GlaxoSmithKline, Intarcia and Sanofi, and personal fees for consultative work from AstraZeneca, Daiichi, Merck Sharp \& Dohme, NovoNordisk and Boehringer-Ingelheim.

\section{Acknowledgements}

SKP, OM and JA acknowledge a grant provided by Royal Brisbane Women Hospital Foundation. Melbourne EpiCentre gratefully acknowledges support from the National Health and Medical Research Council and the Australian Government's National Collaborative Research Infrastructure Strategy (NCRIS) initiative through Therapeutic Innovation Australia. The authors are grateful to all contributors in the CEMR database. OM gratefully acknowledges the PhD scholarship from Queensland University of Technology, Australia, and her co-supervisors Prof. Ross Young and Prof. Louise Hafner of the same university.

\section{Author contributions}

SKP conceived the idea and was responsible for the primary design of the study. OM, JA and JG contributed in the study design. OM conducted the data extraction, and SKP and OM jointly conducted the statistical analyses. The first draft of the manuscript was developed by OM and SKP, and all authors contributed to the finalisation of the manuscript. SKP had full 
access to all the data in the study and is the guarantor, taking responsibility for the integrity of the data and the accuracy of the data analysis. Aggregated data is available upon on request.

\section{$<$ H1 $>$ References}

1 Deacon CF, Mannucci E, Ahrén B. Glycaemic efficacy of glucagon-like peptide- 1 receptor agonists and dipeptidyl peptidase - 4 inhibitors as add- on therapy to metformin in subjects with type 2 diabetes-a review and meta analysis. Diabetes Obes Metab 2012; 14: 762-767.

2 Paul SK, Agbeve J, Maggs D, Best JH. Comparison of trajectories of self-monitored glucose levels by hypoglycemia status over 52 weeks of treatment with insulin glargine or exenatide once weekly. J Diabetes 2016; 8: 148-157.

3 American Diabetes Association. Standards of Medical Care in Diabetes-2018. Diabetes Care 2018; 41(Suppl 1): S4.

4 Paul SK, Klein K, Maggs D, Best JH. The association of the treatment with glucagonlike peptide-1 receptor agonist exenatide or insulin with cardiovascular outcomes in patients with type 2 diabetes: a retrospective observational study. Cardiovasc Diabetol 2015; 14: 10 .

5 Wu S, Cipriani A, Yang Z, Yang J, Cai T, Xu Y et al. The cardiovascular effect of incretin-based therapies among type 2 diabetes: a systematic review and network metaanalysis. Expert Opin Drug Saf 2018; 17: 243-249.

6 Azoulay L, Filion KB, Platt RW, Dahl M, Dormuth CR, Clemens KK et al. Incretin based drugs and the risk of pancreatic cancer: international multicentre cohort study. BMJ 2016; 352: i581.

7 Azoulay L. Incretin-based drugs and adverse pancreatic events: almost a decade later and uncertainty remains. Diabetes Care 2015; 38: 951-953.

8 Tkáč I, Raz I. Combined analysis of three large interventional trials with gliptins indicates increased incidence of acute pancreatitis in patients with type 2 diabetes. Diabetes Care 2017; 40: 284-286.

9 Thomsen RW, Pedersen L, Møller N, Kahlert J, Beck-Nielsen H, Sørensen HT. Incretin-based therapy and risk of acute pancreatitis: a nationwide population-based case-control study. Diabetes Care 2015; 38: 1089-1098. 
10 Faillie J-L, Azoulay L, Patenaude V, Hillaire-Buys D, Suissa S. Incretin based drugs and risk of acute pancreatitis in patients with type 2 diabetes: cohort study. BMJ 2014; 348: g2780.

11 Roshanov PS, Dennis BB. Incretin-based therapies are associated with acute pancreatitis: meta-analysis of large randomized controlled trials. Diabetes Res Clin Pract 2015; 110: e13-e17.

12 Wang T, Wang F, Gou Z, Tang H, Li C, Shi L et al. Using real-world data to evaluate the association of incretin-based therapies with risk of acute pancreatitis: a meta-analysis of 1324515 patients from observational studies. Diabetes Obes Metab $2015 ; 17: 32-41$.

13 Li L, Shen J, Bala MM, Busse JW, Ebrahim S, Vandvik PO et al. Incretin treatment and risk of pancreatitis in patients with type 2 diabetes mellitus: systematic review and meta-analysis of randomised and non-randomised studies. BMJ 2014; 348: g2366.

14 Chou H-C, Chen W-W, Hsiao F-Y. Acute pancreatitis in patients with type 2 diabetes mellitus treated with dipeptidyl peptidase- 4 inhibitors: a population-based nested casecontrol study. Drug Saf 2014; 37: 521-528.

15 Chang C-H, Lin J-W, Chen S-T, Lai M-S, Chuang L-M, Chang Y-C. Dipeptidyl peptidase-4 inhibitor use is not associated with acute pancreatitis in high-risk type 2 diabetic patients: a nationwide cohort study. Medicine 2016; 95: e2603.

16 Knapen L, van Dalem J, Keulemans Y, van Erp N, Bazelier M, De Bruin M et al. Use of incretin agents and risk of pancreatic cancer: a population-based cohort study.

Diabetes Obes Metab 2016; 18: 258-265.

17 Knapen LM, de Jong RG, Driessen JH, Keulemans YC, van Erp NP, De Bruin ML et $a l$. The use of incretin agents and risk of acute and chronic pancreatitis: a population-based cohort study. Diabetes Obes Metab 2017; 19: 401-411.

18 Chen H, Zhou X, Chen T, Liu B, Jin W, Gu H et al. Incretin-based therapy and risk of pancreatic cancer in patients with Type 2 diabetes mellitus: a meta-analysis of randomized controlled trials. Diabetes Ther 2016; 7: 725-742.

19 Centers for Disease Control and Prevention. National Diabetes Statistics Report: Estimates of Diabetes and its Burden in the United States, 2014. Atlanta, GA: US Department of Health and Human Services, 2014.

20 Crawford AG, Cote C, Couto J, Daskiran M, Gunnarsson C, Haas K et al. Comparison of GE Centricity Electronic Medical Record database and National Ambulatory 
Medical Care Survey findings on the prevalence of major conditions in the United States. Popul Health Manag 2010; 13: 139-150.

21 Paul SK, Shaw J, Montvida O, Klein K. Weight gain in insulin-treated patients by body mass index category at treatment initiation: new evidence from real-world data in patients with type 2 diabetes. Diabetes Obes Metab 2016; 18: 1244-1252.

22 ElHafeez SA, Torino C, D'Arrigo G, Bolignano D, Provenzano F, Mattace-Raso F et al. An overview on standard statistical methods for assessing exposure-outcome link in survival analysis (Part II): the Kaplan-Meier analysis and the Cox regression method. Aging Clin Exp Res 2012; 24: 203-206.

23 Rotnitzky A, Robins JM. Inverse probability weighting in survival analysis. Encyclopedia of Biostatistics. Chichester: Wiley, 2005.

24 Austin PC, Stuart EA. The performance of inverse probability of treatment weighting and full matching on the propensity score in the presence of model misspecification when estimating the effect of treatment on survival outcomes. Stat Methods Med Res 2017; 26: 1654-1670.

25 Austin PC. Variance estimation when using inverse probability of treatment weighting (IPTW) with survival analysis. Stat Med 2016; 35: 5642-5655.

26 Pini M, Rhodes DH, Castellanos KJ, Cabay RJ, Grady EF, Fantuzzi G. Rosiglitazone improves survival and hastens recovery from pancreatic inflammation in obese mice. PLoS One 2012; 7: e40944.

27 Wan H, Yuan Y, Liu J, Chen G. Pioglitazone, a PPAR- $\gamma$ activator, attenuates the severity of cerulein-induced acute pancreatitis by modulating early growth response-1 transcription factor. Transl Res 2012; 160: 153-161.

28 Woodcock J, Sharfstein JM, Hamburg M. Regulatory action on rosiglitazone by the US Food and Drug Administration. N Engl J Med 2010; 363: 1489-1491.

29 Forsmark CE, Vege SS, and Wilcox CM. Acute pancreatitis. N Engl J Med 2016; 375: 1972-1981.

FIGURE 1. Flow-chart of the study cohort. EMR, electronic Medical Record; DDP-4i, dipeptidyl peptidase-4 inhibitor; GLP-1RA, glucagon-like peptide-1 receptor agonist; INS, insulin; SU, sulfonylurea; TZD, thiazolidinedione

\section{$<$ H1 >Supporting Information}

This article is protected by copyright. All rights reserved 
Additional Supporting Information may be found in the online version of this article:

Table S1. Weighted standardized differences in balanced groups.

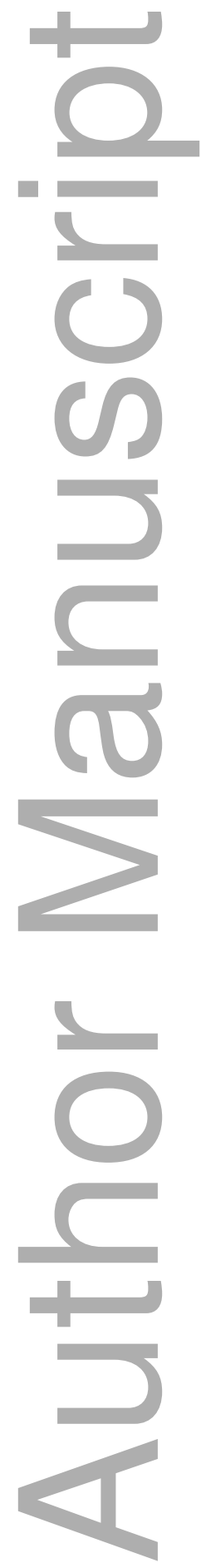


Table 1. Basic characteristics at the time of second-line anti-hyperglycaemic therapy initiation (index date), and the number of events post index date

\begin{tabular}{|c|c|c|c|c|c|c|}
\hline 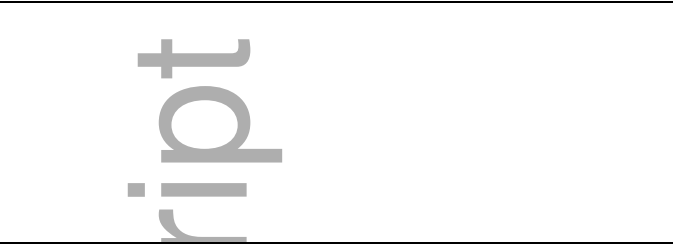 & $\begin{array}{l}\text { Dipeptidyl } \\
\text { peptidase-4 } \\
\text { inhibitor }\end{array}$ & $\begin{array}{l}\text { Glucagon-like } \\
\text { peptide-1 } \\
\text { receptor } \\
\text { agonist }\end{array}$ & Insulin & Sulfonylurea & Thiazolidinedione & Total \\
\hline $\mathrm{N}$ & 50095 & 12654 & 34805 & 110747 & 17597 & 225898 \\
\hline Age, years* & $58(12)$ & $53(12)$ & $57(13)$ & $60(12)$ & $59(12)$ & $59(12)$ \\
\hline Men $\dagger$ & $24034(48)$ & 4346 (34) & $16302(47)$ & $57876(52)$ & $9174(52)$ & 111732 (49) \\
\hline White European ancestry $\dagger$ & $34989(70)$ & $9613(76)$ & $23229(67)$ & $76430(69)$ & $11791(67)$ & $156052(69)$ \\
\hline Black $\dagger$ & $5852(12)$ & $1083(9)$ & $5083(15)$ & $12971(12)$ & $1581(9)$ & $26570(12)$ \\
\hline Current tobacco use $\dagger$ & $4872(10)$ & $979(8)$ & $3929(11)$ & $9980(9)$ & $797(5)$ & $20557(9)$ \\
\hline Former tobacco use $\dagger$ & $8086(16)$ & $1989(16)$ & $5729(16)$ & $16790(15)$ & $1232(7)$ & $33826(15)$ \\
\hline Never tobacco use $\dagger$ & $15265(30)$ & $3839(30)$ & $8828(25)$ & $26797(24)$ & $2482(14)$ & $57211(25)$ \\
\hline Tobacco use - unknown status $\dagger$ & $21872(44)$ & $5847(46)$ & $16319(47)$ & $57180(52)$ & $13086(74)$ & $114304(51)$ \\
\hline Diabetes duration prior to index date, months* & $14.35(21.47)$ & $13.50(20.85)$ & $7.76(16.61)$ & $11.13(19.92)$ & $6.34(14.27)$ & $11.09(19.64)$ \\
\hline Treatment duration, months* & $26.22(20.07)$ & $24.85(20.34)$ & $31.15(24.77)$ & $31.59(24.94)$ & $32.49(26.13)$ & $30.03(23.91)$ \\
\hline Follow-up, yearst & $2.54(1.3,4.11)$ & $\begin{array}{l}2.67(1.25, \\
4.67)\end{array}$ & $\begin{array}{l}2.27(1.13 \\
3.99)\end{array}$ & $2.67(1.31,4.6)$ & $4.33(1.99,6.79)$ & $\begin{array}{l}2.66(1.3, \\
4.54)\end{array}$ \\
\hline Follow-up, years* & $2.91(1.96)$ & $3.24(2.39)$ & $2.81(2.13)$ & $3.24(2.37)$ & $4.56(2.89)$ & $3.20(2.34)$ \\
\hline $\mathrm{HbA}_{1 \mathrm{c}}, \mathrm{mmol} / \mathrm{mol} \uparrow$ & $61(52,74)$ & $56(48,68)$ & $74(56,93)$ & $63(53,77)$ & $55(48,68)$ & $62(52,78)$ \\
\hline $\mathrm{HbA}_{1 \mathrm{c}}, \% \ddagger$ & $7.7(6.9,8.9)$ & $7.3(6.5,8.4)$ & $8.9(7.3,10.7)$ & $7.9(7.0,9.2)$ & $7.2(6.5,8.4)$ & $7.8(6.9,9.3)$ \\
\hline $\mathrm{SBP}, \mathrm{mmHg} *$ & $130(14)$ & $128(13)$ & $131(16)$ & $132(16)$ & $130(15)$ & $131(15)$ \\
\hline $\mathrm{LDL}, \mathrm{mmol} / \mathrm{l}^{*}$ & $2.53(0.91)$ & $2.48(0.88)$ & $2.53(0.96)$ & $2.53(0.91)$ & $2.51(0.88)$ & $2.53(0.91)$ \\
\hline Triglicerides, mmol/l & $\begin{array}{l}1.66(1.22 \\
2.21)\end{array}$ & $\begin{array}{l}1.68(1.22, \\
2.24)\end{array}$ & $\begin{array}{l}1.61(1.15, \\
2.20)\end{array}$ & $1.67(1.22,2.24)$ & $1.55(1.12,2.11)$ & $\begin{array}{l}1.65(1.21 \\
2.21)\end{array}$ \\
\hline
\end{tabular}

This article is protected by copyright. All rights reserved 


\begin{tabular}{|c|c|c|c|c|c|c|}
\hline Weight, $\mathrm{kg}^{*}$ & $98(24)$ & $108(26)$ & $100(26)$ & $97(25)$ & $99(24)$ & $99(25)$ \\
\hline BMI, $\mathrm{kg} / \mathrm{m}^{2} *$ & $34.4(7.6)$ & $38.2(83)$ & $35.2(8.3)$ & $34.1(7.7)$ & $34.6(7.9)$ & $34.6(7.9)$ \\
\hline Obese & $32567(70)$ & $10201(87)$ & $22600(71)$ & $67899(68)$ & $10763(70)$ & $144030(70)$ \\
\hline Hypertension $\dagger$ & $28063(56)$ & $6675(53)$ & $17477(50)$ & $60207(54)$ & $8434(48)$ & $120856(54)$ \\
\hline Cardiovascular disease $\dagger$ & $8796(18)$ & $1531(12)$ & $7745(22)$ & $22995(21)$ & $2958(17)$ & $44025(19)$ \\
\hline Chronic kidney disease $\dagger$ & $1525(3)$ & $229(2)$ & $1129(3)$ & $3910(4)$ & $447(3)$ & $7240(3)$ \\
\hline Received third anti-hyperglycaemic agent $\dagger$ & $23318(47)$ & $5986(47)$ & $4482(13)$ & $34905(32)$ & $7015(40)$ & $75706(34)$ \\
\hline Received cardio-protective medication $\dagger$ & $46395(93)$ & $11336(90)$ & $31862(92)$ & $103553(94)$ & $16390(93)$ & $209536(93)$ \\
\hline Received non-steroidal anti-inflammatory drugs & $37265(74)$ & $9269(73)$ & $25121(72)$ & $82963(75)$ & $13207(75)$ & $167825(74)$ \\
\hline Received anti-hypertensive $\dagger$ & $3613(7)$ & $824(7)$ & $3821(11)$ & $10518(10)$ & $1627(9)$ & $20403(9)$ \\
\hline Sub-cohort $1 \uparrow$ & $49419(99)$ & $12518(99)$ & $34098(98)$ & $108553(98)$ & $17294(98)$ & $221882(98)$ \\
\hline Sub-cohort $2 \dagger$ & $31618(63)$ & $7580(60)$ & $18924(54)$ & $64086(58)$ & $9274(53)$ & $131482(58)$ \\
\hline
\end{tabular}

Values are given as *mean (SD), †n (\%) or \$median (IQR).

Sub-cohort 1, patients from the study cohort excluding those with acute pancreatitis, other disease of pancreas or any type of cancer within 6 months of index date.

Sub-cohort 2, patients from the study cohort with non-missing $\mathrm{HbA}_{1 \mathrm{c}}$ measure at index date.

1

Table 2. Event rates (95\% CI) per 1000 person-years; adjusted mean time to events (95\% CI) in dipeptidyl peptidase-4 (DPP-4) group and adjusted difference in time to events in other treatment groups with DPP-4 inhibitor as a reference

\begin{tabular}{|c|c|c|c|}
\hline 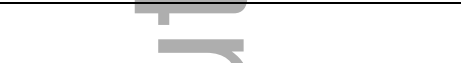 & Acute pancreatitis $(95 \% \mathrm{CI})$ & Cancer of pancreas $(95 \% \mathrm{CI})$ & Other disease of pancreas $(95 \% \mathrm{CI})$ \\
\hline \multicolumn{4}{|l|}{ DPP-4 } \\
\hline Rate per 1000 person-years & $1.38(1.21,1.59)$ & $0.46(0.36,0.59)$ & $0.93(0.78,1.10)$ \\
\hline Mean time to event (years) & $2.63(2.38,2.88)$ & $2.70(2.19,3.21)$ & $2.73(2.33,3.12)$ \\
\hline Glucagon-like peptide- 1 receptor & & & \\
\hline
\end{tabular}

This article is protected by copyright. All rights reserved 


\begin{tabular}{|c|c|c|c|}
\hline \multicolumn{4}{|l|}{ agonist } \\
\hline Rate per 1000 person-years & $1.49(1.16,1.92)$ & $0.17(0.08,0.36)$ & $0.78(0.55,1.10)$ \\
\hline Time difference (years) & $-0.18(-0.72,0.37)$ & $3.00(0.84,5.16)^{*}$ & $0.52(-0.60,1.65)$ \\
\hline \multicolumn{4}{|l|}{ Insulin } \\
\hline Rate per 1000 person-years & $2.01(1.75,2.31)$ & $0.59(0.46,0.77)$ & $1.48(1.26,1.75)$ \\
\hline Time difference (years) & $-0.48(-0.90,-0.06)^{*}$ & $-0.70(-1.56,0.17)$ & $-0.49(-1.01,0.03)$ \\
\hline \multicolumn{4}{|l|}{ Sulfonylurea } \\
\hline Rate per 1000 person-years & $1.45(1.33,1.58)$ & $0.55(0.47,0.63)$ & $1.04(0.94,1.15)$ \\
\hline Time difference (years) & $-0.01(-0.51,0.50)$ & $-0.57(-1.26,0.11)$ & $-0.43(-1.13,0.28)$ \\
\hline \multicolumn{4}{|l|}{ Thiazolidinedione } \\
\hline Rate per 1000 person-years & $0.89(0.70,1.12)$ & $0.36(0.25,0.52)$ & $0.85(0.67,1.08)$ \\
\hline Time difference (years) & $-0.25(-0.56,0.05)$ & $-0.09(-0.74,0.56)$ & $-0.28(-0.74,0.18)$ \\
\hline
\end{tabular}

$\dagger$ Analyses were balanced on age and the follow-up time by treatment groups, and were adjusted for age, sex, BMI, smoking status and diabetes duration

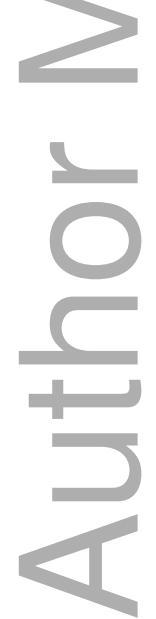

This article is protected by copyright. All rights reserved 


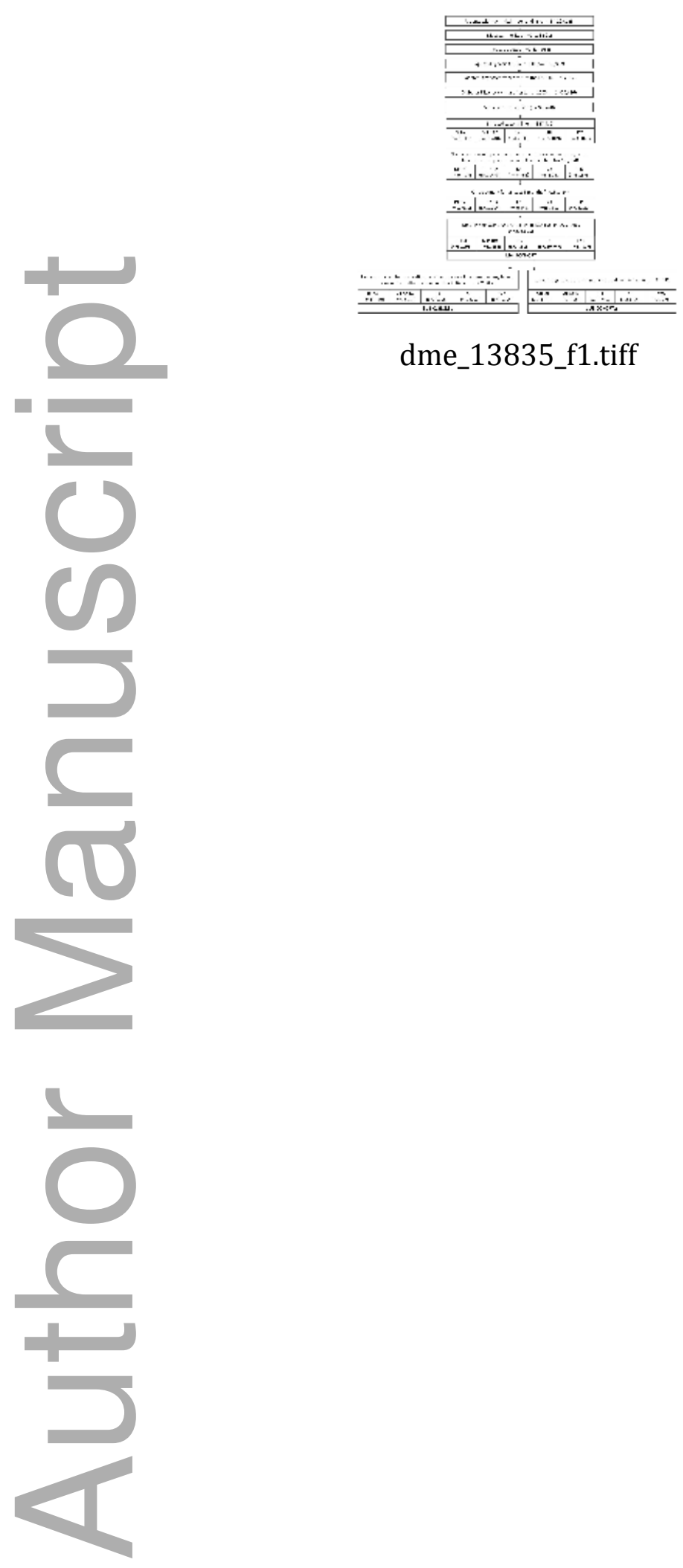

This article is protected by copyright. All rights reserved 


\section{University Library}

\section{- M I N E R VA A gateway to Melbourne's research publications}

Minerva Access is the Institutional Repository of The University of Melbourne

Author/s:

Montvida, O;Green, JB;Atherton, J;Paul, SK

Title:

Treatment with incretins does not increase the risk of pancreatic diseases compared to older anti-hyperglycaemic drugs, when added to metformin: real world evidence in people with Type 2 diabetes

Date:

2019-04-01

Citation:

Montvida, O., Green, J. B., Atherton, J. \& Paul, S. K. (2019). Treatment with incretins does not increase the risk of pancreatic diseases compared to older anti-hyperglycaemic drugs, when added to metformin: real world evidence in people with Type 2 diabetes. DIABETIC MEDICINE, 36 (4), pp.491-498. https://doi.org/10.1111/dme.13835.

Persistent Link:

http://hdl.handle.net/11343/284593 